\title{
Scanning Electrochemical Microscopy-Atomic Force Microscopy Probes from Pyrolyzed Parylene C
}

\author{
Kirstin C. Morton, Maksymilian A. Derylo, Anna E. Weber and Lane A. Baker \\ Department of Chemistry, Indiana University, Bloomington, IN 47405, USA
}

Scanning electrochemical microscopy-atomic force microscopy (SECM-AFM) is an advanced hybrid technique that combines the electrochemical sensitivity of scanning electrochemical microscopy (SECM) with the superior spatial resolution of atomic force microscopy (AFM) by integration of an ultramicroelectrode into an AFM probe[1]. In this technique, surface topography can be measured and electrochemical information collected simultaneously. In this type of experiment, as illustrated in the scheme in Figure 1, the SECM-AFM probe is the working electrode and an $\mathrm{Ag} / \mathrm{AgCl}$ wire serves as the reference electrode. A potential is applied between the working and reference electrode that is sufficient to oxidize or reduce a species of interest in solution as the probe operates in contact-AFM mode. A number of SECM-AFM probes, which contain gold [2], platinum [1], carbon nanotubes [3], and borondoped diamond $[4,5]$ as electrode material, have been demonstrated previously. An area that SECMAFM has yet to be applied is for the detection of neurological species in situ. For application in biological measurements, a carbonaceous electrode material would be advantageous over noble metal materials due to the wide-potential window available, low propensity to biofoul and chemical inertness. As a first step towards this goal, we describe fabrication of SECM-AFM probes from pyrolyzed parylene C (PPC), a conductive carbon material recently shown as a viable AFM probe coating [6], via focused ion beam milling. Focused ion beam parameters have been optimized and probes have been characterized with scanning electron microscopy (SEM), cyclic voltammetry and scanning transmission electron microscopy (STEM). Scanning electron microscopy images, shown in Figure 2, detail a side and top-down view of an FIB-milled PPC SECM-AFM probe. In addition, the resolution of the fabricated probes in liquid will be shown. Also, the effect of gallium ion implantation and damage, as a result of FIB-milling, will be discussed with data from x-ray photoelectron studies.

Electrochemical and spatial diffusion measurements of model neurotransmitter, dopamine hydrochloride, through a porous, synthetic poly(imide) (PI) membrane with PPC SECM-AFM probes will be presented. Measurement of free diffusion of dopamine through a PI membrane serves as a model system to characterize the electrochemical properties of fabricated PPC SECM-AFM probes and to further realization of in situ dopamine measurements in cultured PC12 cells, a model cellular system [7]. 
References:

[1] J. V. Macpherson,P. R. Unwin, Anal. Chem. 72 (2000), p. 276.

[2] M. A. Derylo, et al., Langmuir (2011), p.

[3] D. P. Burt, et al., Nano Lett. 5 (2005), p. 639.

[4] W. Smirnov, et al., Anal. Chem. 83 (2011), p. 4936.

[5] A. Avdic, et al., Nanotechnology 22 (2011), p.

[6] K. C. Morton, et al., J. Electrochem. Soc. 159 (2012), p. H662.

[7] The authors acknowledge funding from the Research Corporation for Scientific Advancement, Cottrell Scholars Award and the National Science Foundation CHE Electrochemistry and Surface Chemistry, Award Number 0847342. Prof. Anne Prieto (Department of Psychological and Brain Sciences, Indiana University) and Mr. Rahul Thakar are greatfully thanked for their supply and help with culture of PC12 cells

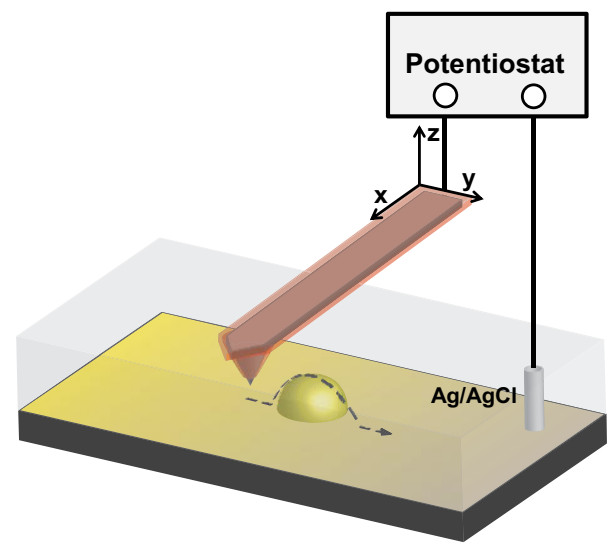

Figure 1. A scheme of a scanning electrochemical microscopy-atomic force microscopy (SECM-AFM) experimental set-up. A two-electrode system is used, in which the AFM probe is the working electrode (WE) and the $\mathrm{Ag} / \mathrm{AgCl}$ wire is the reference electrode (RE). Both electrodes are immersed in a liquid solution containing a redox probe of interest. A potential is applied between the WE and RE to oxidize or reduce redox molecules. A two map of spatial oxidation or reduction current differences is generated as the working electrode scans over the surface. Since the electrode is close to the AFM tip apex, topography and current images are correlated.
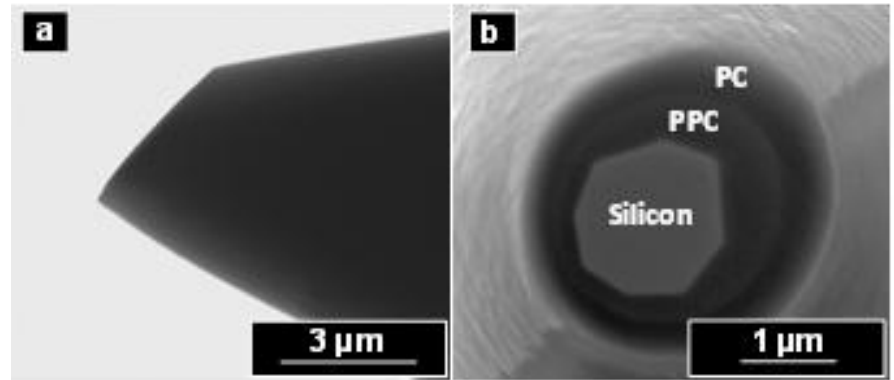

Figure 2. Side (a) and top-down (b) scanning transmission electron microscopy images of an FIBmilled, pyrolyzed parylene C (PPC) scanning electrochemical microscopy-atomic force microscopy (SECM-AFM) probe. The silicon (interior octagon), PPC ring-electrode (inner ring) and parylene C (PC) insulation layer (outer ring) are visible and labeled in the image. 